\title{
Zuber's Ten Principles of Patient- Centric Patient Experience and a Framework
}

\author{
Zuber Mujeeb Shaikh \\ Director, Corporate Quality Improvement, Patient Safety and Risk Management \\ Dr. Sulaiman Al-Habib Medical Group, \\ Riyadh-11643, Kingdom of Saudi Arabia \\ ORCID-ID: 0000-0002-1491-8379 \\ Email: drzuber5@yahoo.co.in
}

Area/Section: Health Sciences.

Type of the Paper: Review Paper.

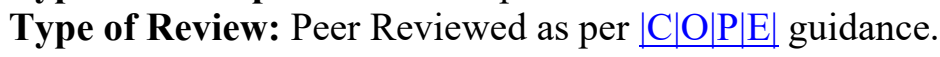

Indexed in: OpenAIRE.

DOI: http://doi.org/10.5281/zenodo.4517634

Google Scholar Citation: $\underline{\text { IJHSP }}$

How to Cite this Paper:

Shaikh, Zuber Mujeeb, (2021). Zuber's Ten Principles of Patient- Centric Patient Experience and a Framework. International Journal of Health Sciences and Pharmacy (IJHSP), 5(1), 110. DOI: http://doi.org/10.5281/zenodo.4517634

International Journal of Health Sciences and Pharmacy (IJHSP)

A Refereed International Journal of Srinivas University, India.

(C) With Author.

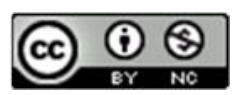

This work is licensed under a Creative Commons Attribution-Non-Commercial 4.0 International License subject to proper citation to the publication source of the work.

Disclaimer: The scholarly papers as reviewed and published by the Srinivas Publications (S.P.), India are the views and opinions of their respective authors and are not the views or opinions of the SP. The SP disclaims of any harm or loss caused due to the published content to any party. 


\title{
Zuber's Ten Principles of Patient- Centric Patient Experience and a Framework
}

\author{
Zuber Mujeeb Shaikh \\ Director, Corporate Quality Improvement, Patient Safety and Risk Management \\ Dr. Sulaiman Al-Habib Medical Group, \\ Riyadh-11643, Kingdom of Saudi Arabia \\ ORCID-ID: https://orcid.org/0000-0002-1491-8379 \\ Email: drzuber5@yahoo.co.in
}

\begin{abstract}
A patient-centric patient experience is a key to high-quality healthcare service industry since; it has been observed that the patients with good experience add trust, cohesiveness with treating the healthcare team and a better continuity of care, which leads to a better outcome and excels patient experience. Objective: To develop a patient-centric patient experience framework. Method: This is a review of literature study and the data were collected with comprehensive searches in the online databases of goggle scholars and research gate. Conclusions: The study concluded with "Zuber's ten principles of patient-centric patient experience and a framework."
\end{abstract}

Keywords: Patient-Centric, Patient Experience, Framework, Accreditation, Quality, Zuber

\section{INTRODUCTION :}

Patient-centric care is defined as "Putting the patient first in an open and sustained engagement of the patient to respectfully and compassionately achieve the best experience and outcome for that person and their family."

The healthcare service industry is moving toward a patient-centric patient experience, in which the patient is at the center and the healthcare service providers provide personalized care in all aspects, which include but are not limited to:

a) Care of patient and their families

b) Respect patients' needs, preferences, values and beliefs,

c) Coordination of administrative (frontline, billing etc.), clinical, ancillary and support care

d) Provide continuous education or information and involve in care the patient and family

e) Provide soothe environment with no pain and good living

f) Provide support during emotional issues

In this modern era of science, technology and quality, the healthcare service industry is a thriving sector, which is continuously on high demand in all continents of the world.

Patient experience is defined as "To assess patient experience, one must determine from patients whether something that should happen in a healthcare setting (such as clear communication with a provider) happened or how often it happened" (Agency for Healthcare Research and Quality -AHRQ).

The concept of patient satisfaction is since the 1960s, but there was no research conducted actively until 1970s and however the research scholars initiated active research in the early 1980s and replaced the idea of "quantity of life" with a more patient-centered concept of quality of life (Ekram S, Rahman, 2006) [1].

\section{REVIEW OF LITERATURE :}

Researchers had revealed that accreditation showed positive impact on hospital emergency transport services (Shaikh, 2016) [2], Department Services (Shaikh, 2017) [3], Dietary Services (Shaikh, 2017) [4], Laboratory Department Services (Shaikh, 2017) [5], Emergency Department Services (Shaikh, 2017) [6], In-Patient Department Services (Shaikh, 2017) [7], Hemodialysis Department Services (Shaikh, 2017) [8], Radiology 
Department Services (Shaikh, 2017) [9], Pharmacy Department Services (Shaikh, 2017) [10], Out-Patient Department (Shaikh, 2017) [11].

Researchers had conducted several studies earlier to understand the impact of hospital accreditation on the patients' experience. It has been proved that the accreditation has a positive impact on the patients' experience of hospital Emergency Transport Services (Shaikh, 2018) [12], Hemodialysis services (Shaikh, 2019) [13] and Emergency Department Services, (Shaikh, 2019) [14].

The accreditation of hospitals revealed improvement in the Human Resources Department Services (Shaikh, 2017) [15], Occurrence Variance Report or Incident Reports system (Shaikh, 2018) [16] and Laboratory \& Blood Bank performance (Shaikh, 2018) [17], Critical Care Unit Outcome Quality Measures, (Shaikh, AlOmari, A., \& Ahmed, 2018) [18].

The Planetree Person Centered Care accreditation of an accredited (national and international) hospital revealed a positive impact on its services (Shaikh, Al-Omari, \& Ahmed, 2018) [19], and Person-Centered Care Design has a positive impact on the healing process [20].

The researchers have revealed that the accreditation has improved patient satisfaction, experience and outcomes [21, 22, 23, 24, 25, 26, 27, 28, 29, 30, 31, 32, 33, 34, 35, 36, 37, 38, 39, 40, 41, 42, 43, 44, 45, 46, $47,48,49]$.

\section{ZUBER'S TEN PRINCIPLES OF PATIENT-CENTRIC PATIENT EXPERIENCE AND A FRAMEWORK :}

Dr. Zuber has developed "Ten Principles of Patient- Centric Patient Experience and A Framework".

Zuber's Ten Principles of Patient- Centric Patient Experience are as follows:

1) Governance and Leadership

2) Innovative Technology and Tools

3) Hospital Environment

4) Management of Information, Communication and Education

5) Staff Engagement and Empowerment

6) Patient Experience Culture

7) Access, Transition and Continuity of Care

8) Organized and Incorporated Care

9) Patient-Centric Care

10) Quality Improvement

Below are the components with details and explanations of Zuber's Ten Principles of Patient- Centric Patient Experience:

1) Governance and Leadership:

a) Include Patient Experience in the Board of Governance Bylaws and its reports.

b) Include Patient Experience in the hospital Strategic Plan, Operational Plan and Departmental Operational Plans.

c) Provide all required resources (human, capital, materials-supplies, furniture, and technological resources' etc.) for Patient Experience.

d) Conduct the patient, family, staff, stakeholder and community need assessment for patient experience.

e) Establish an organizational structure for patient experience; create specific positions, job descriptions for assigned personnel.

f) Provide continuous education and training for leaders and staff to encourage an organizational culture of patient experience.

g) Develop education and training materials and resources in national and English. 
h) Ensure that leadership is visible and accessible in the organization and routinely engaged with patients and frontline staff.

i) Embed patient experience in all aspects of leadership development.

j) Conduct the leadership rounds monthly and on weekends, night shifts and on holidays as a surprise leadership round.

k) Implement award, appreciation and recognition program for patient experience.

1) Establish and implement policies for all barriers to improve access and communication with the patient, family, staff, stakeholder and community leaders.

m) Support in implementing the concept of Patient Friendly Hospital.

n) Exemplify patient experience performance by incorporating in mission and vision.

o) Implement and monitor compliance to the Bill of Patients Rights and Responsibilities.

p) Respect the religious needs, values and beliefs of the patients, their families and carers fair and equally.

q) Provide education and training on Patient Experience to all staff.

r) Conduct the patient experience surveys by "Mystery Shoppers."

s) Celebrate the identified good practices monthly.

t) Involve patients, their families and carers in the assessment and appraisal processes for staff.

u) Respect, engage, collaborate and make accountable, shared commitment,

v) Develop policies, procedures and plans on Patient Experience by a collaborative method and by incorporating the mission, vision and values.

w) Educate all staff on the approved policies, procedures and plans on Patient Experience and continuously monitor the compliance for improvement.

x) Provide consistent and expert care all days a week by safe hands.

y) Endorse health and happiness for patients, families, carers and staff.

z) Force change at all levels to give importance for improvement.

2) Hospital Environment:

a) Design a supportive physical, technological, and psychosocial setting that forms a comfortable atmosphere for clinical and procedural processes to generate quality outcomes.

b) Provide clear signages for all services by using an international healthcare signage and symbols.

c) Ensure that the facility is safety for physically or differently abled persons visiting to hospital for care, treatment, etc.

d) Ensure that there are no physical or other barriers for access and communication.

3) Management of Information, Communication and Education:

a) Provide on-demand advice, diagnosis, and treatment for minor health conditions.

b) Provide open notes and explanation or shared medical records.

c) Ensure how well healthcare providers communicate with patients.

d) Provide continues information to patient, their families and carers on clinical conditions, improvement, diagnosis, outcome, care process.

e) Provide training to advanced physicians' communication skills.

f) Support groups and self-care.

g) Implement a reminder system for immunization and preventive services.

h) Establish ways of communications in order to receive more ideas on patient experience from staff, patients, families and carers.

i) Promote information transparency and symmetry by giving patent and provider access to the same data and knowledge.

j) Ensure that healthcare workers talk with patients, their families and carers about their patients' health.

k) Provide disease-specific resources to patients or their families.

1) Provide information about the cost of care and treatment.

4) Staff Engagement and Empowerment:

a) Empower all full-time, part-time, locum, trainees, and students etc. to improve patient's experience by change management and make them accountable. 
b) Empower all full-time, part-time, locum, trainees, and students etc. to gather feedback from patients, their families and carers to improve patient's experience by change management.

c) Establish a mechanism to collect the real time patient's experience feedback.

5) Innovative Technology and Tools:

a) Implement innovative technology for conducting online patient experience surveys in all departments of the hospital, education and training of staff, patients and their families and carers.

b) Ensure that technology and tools are efficiently used for expanding capacities and extending boundaries of care.

6) Patient Experience Culture:

a) Establish a culture in which staffs are proud to work for the organization and speak highly of the culture.

b) Empower the staff.

c) Create a patient friendly hospital culture.

d) Establish a process to identify and celebrate achievements of staff that consistently exceed patient expectations.

e) Develop a mechanism of collecting the patients' stories.

f) Publish the patient stories on the hospital portal after taking the written consent of the patient by maintaining the national laws and regulations.

g) Ensure that all staff is helpful, courteous, and respectful.

h) Assist in getting information in a needed language or format.

i) Cultivate cultural competence.

j) Collaborate help, understand, personalize and manager patients' needs and care.

7) Access, Transition and Continuity of Care:

a) Assist in getting timely care, appointments, and information between visit communications.

b) Assist in providing care on timeliness and openness.

c) Assist in getting information about the health plan and cost of care.

d) Establish quality measures to be monitored on patient experience.

e) Provide rapid internal and external referrals.

f) Assist patients, their families and carers in planned and unplanned visits, getting needed and quick care and getting access to specialists.

g) Provide internet access for health information and advice.

h) Assist in providing care based on the identified needs during transition.

i) Involve the patients, their families and carers whenever there is a change in the level of care within the organization or transferred out for treatment and care.

j) Communicate with patients, their families and carers after leaving medical advice, discharged against medical advice, left against medical advice and transferred to another healthcare facility.

k) Conduct the patients, their families and carers feedback on ambulance services use for transfers.

8) Organized and Incorporated Care:

a) Provide tools to help patients, families and carers to communicate their needs.

b) Ensure that the healthcare workers provided information is useful to coordinate patient care.

c) Ensure that well doctors coordinate care and keep patients informed.

d) Provide coordinated, integrated care across the care of continuum.

e) Involve the patients, their families and carers in the care decisions, treatment, and discharge process.

f) Communicate with patients, their families and carers after discharges.

g) Remind and communicate with patients, their families and carers after discharge about their planned visits, treatment, medication refills, procedures and tests, etc.

9) Patient-Centric Care:

a) Give patient locus for control of care decisions by using information transparency and communication to enhance knowledge and participation. 
b) Develop, implement and evaluate the action plan prepared based on the feedback received from patients, their families and carers.

c) Implement the concept of no decision about me without me.

d) Establish a culture of respecting patient's ethics, choices, requirements, cultural issues, self-esteem, solitude and autonomy of patients and service users.

e) Improve quality of-life issues of the patients.

f) Provide physical comfort, clean and comfortable surroundings.

g) Ensure that pain is managed and education is provided to the patients, their families and carers.

h) Help with activities of daily living.

i) Implement Person Centered Care Competencies for all relevant staff.

j) Conduct the feedback for food services.

k) Implement Teach Me, Ask Me 3 Concepts.

1) Implement arts and entertainment Program.

10) Quality Improvement:

a) Capture the data for monitoring the effectiveness of Patient Experience Framework.

b) Establish a system to capture the real time feedback of patients and families.

c) Prioritize quality outcomes to maximize value and consistently prioritize good clinical results.

d) Establish an accessible user-friendly complaints process and to monitor the compliance and outcomes.

e) Publish the Patient Experience Quality Measures on the website of the hospital once data are validated and approved by the hospital management.

f) Submit the reports monthly to the hospital management and to the Board of Governance on Patient Experience and feedback.

g) Publish the Patient Experience and feedback in the hospital newsletters for the staff information.

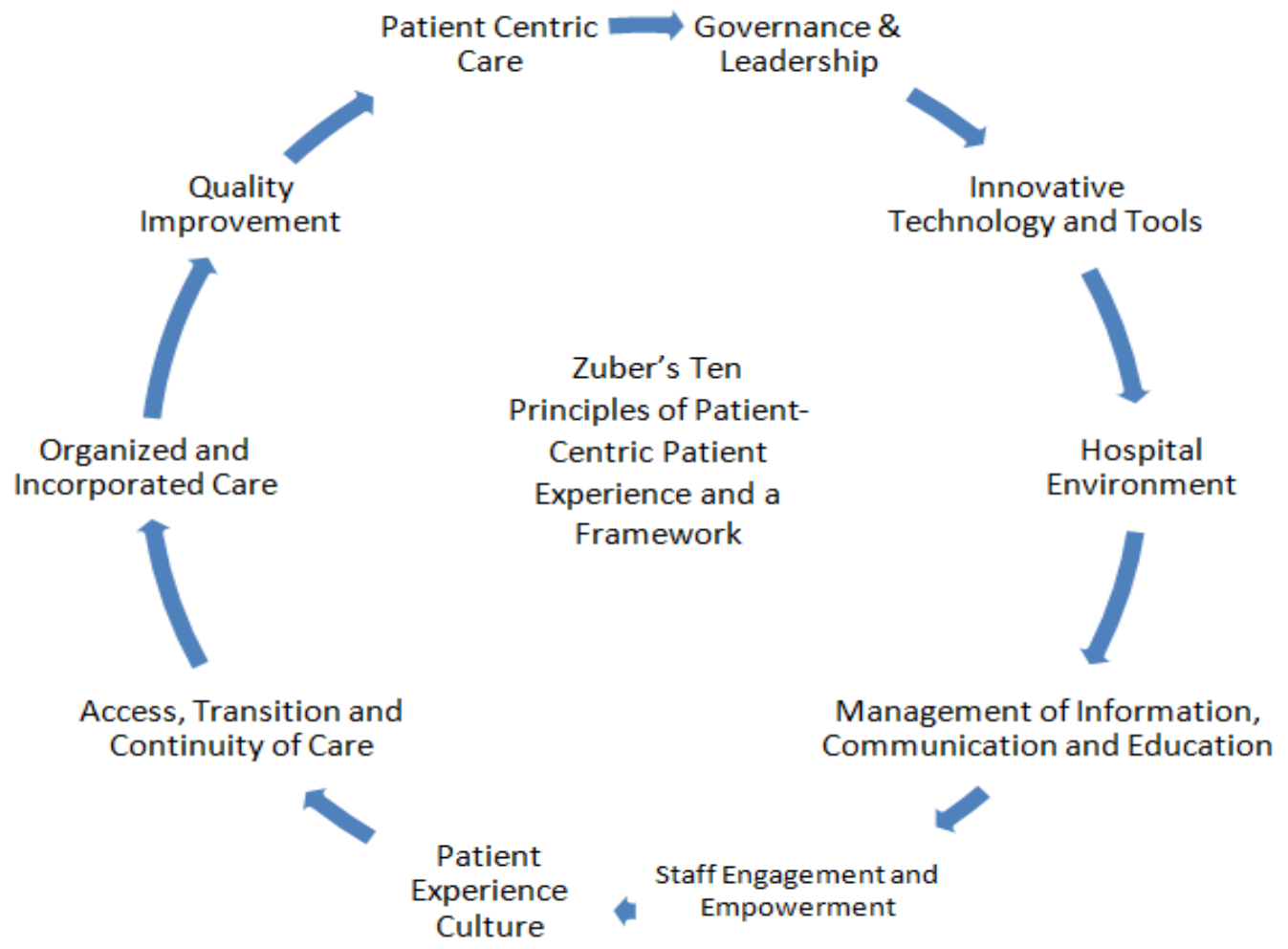

Fig. 1: Zuber's Patient-Centric Patient Experience Framework 


\section{FURTHER RESEARCH :}

Further research is needed to understand the impact of Zuber's ten principles of patient-centric patient experience and framework on the patient experience.

\section{PRACTICE IMPLICATIONS :}

The healthcare providers should implement Zuber's ten principles of patient-centric patient experience and framework for improving patient's experience.

\section{CONCLUSION :}

The study concluded in a new concept and framework "Zuber's ten principles of patient-centric patient experience and framework."

\section{LIMITATIONS OF THE STUDY :}

Patient-centric patient experience is the only limitation of this study.

\section{DIRECTIONS FOR FUTURE RESEARCH :}

In the future, research should be conducted to study the overall impact of Zuber's ten principles of patientcentric patient experience and framework in all clinical, support and ancillary service areas of the hospital.

\section{SOURCES OF FUNDING FOR THE STUDY :}

This study was self-funded by the author.

\section{DISCLAIMER :}

This publication contains information obtained from authentic and highly regarded sources. Reasonable effort has been made to publish reliable data and information, but the author and publisher cannot assume responsibility for the validity of all materials or for the consequences of the use.

All rights reserved. No part of this publication may be reproduced, stored in a retrieval system or transmitted, in any form, or by any means, electronic, mechanical, photocopying, recording or otherwise, without permission, in writing, from the publisher or the author.

\section{REFERENCES :}

[1] Ekram S, Rahman F. (2006). The concept of patient satisfaction as it relates to contemporary health care. TAJ: Journal of Teachers Association, 19(1), 1-2.

[2] Shaikh, Z. M. (2016). The impact of hospital accreditation on the ambulance services satisfaction. International Journal of Emerging Research in Management \& Technology (IJERMT), 5(12), 76-84.

[3] Shaikh, Z. (2017). The Impact of Hospital Accreditation on the Patient's Satisfaction of physical therapy Department Services. International Journal of Business, Management and Allied Sciences (IJBMAS), 4(4.2017), 143-154.

[4] Shaikh, Z. M. (2017). The Impact of Hospital Accreditation on the Patients Satisfaction of Dietary Services. International Journal of Business, Management and Allied Sciences (IJBMAS), 4(4), 1-12.

[5] Shaikh, Z. M. (2017). The Impact of Hospital Accreditation on the Patients Satisfaction of Laboratory Department Services. International Journal of Business, Management and Allied Sciences (IJBMAS), 4(2), 4277-4289.

[6] Shaikh, Z. M. (2017). The Impact of Hospital Accreditation on the Patients Satisfaction of Emergency Department Services. International Journal of Business, Management and Allied Sciences (IJBMAS), 4(3), 4330-4339.

[7] Shaikh, Z. M. (2017). The Impact of Hospital Accreditation on the Patient Satisfaction of In-Patient Department Services. International Journal of Emerging Research in Management \& Technology 
(IJERMT), 6(8), 368-383.

[8] Shaikh, Z. M. (2017). The Impact of Hospital Accreditation on the Patients Satisfaction of Haemodialysis Department Services. International Journal of Emerging Research in Management \& Technology (IJERMT), 6(8), 384-392.

[9] Shaikh, Z. M. (2017). The Impact of Hospital Accreditation on the Patients Satisfaction of Radiology Department Services. International Journal of Business, Management and Allied Sciences (IJBMAS), $4(1), 4120-4130$.

[10] Shaikh, Z. M. (2017). The Impact of Hospital Accreditation on the Patient's Satisfaction of Pharmacy Department Services. International Journal of Business, Management and Allied Sciences, 4(4), 189199.

[11] Shaikh, Z. M. (2017). The Impact of Hospital Accreditation on the Patients Satisfaction of Out-Patient Department Services. International Journal of Business, Management and Allied Sciences (IJBMAS), 4(3), 4384-4398.

[12] Zuber Mujeeb Shaikh (2018). Impact of National Accreditation on the Patients' Experience of Ambulance Services: A Case Study. RESEARCH REVIEW International Journal of Multidisciplinary, 3(08), 177-181.

[13] Zuber Mujeeb Shaikh. (2019). The Impact of Hospital Accreditation on the Patients' Experience of Hemodialysis Department: A Case Study. International Journal of Health Sciences and Pharmacy (IJHSP), 3(1), 31-39.

[14] Zuber Mujeeb Shaikh. (2019). The Impact of Hospital Accreditation on the Patients' Experience of Emergency Department: A Case Study. RESEARCH REVIEW International Journal of Multidisciplinary, 4(2), 803-810.

[15] Shaikh, Z. M. (2017). The Impact of Hospital Accreditation on the Completeness of Personnel Files in Human Resource Department. International Journal of Business, Management and Allied Sciences (IJBMAS), 4(4), 236-244.

[16] Shaikh, Z. M. (2018). The Impact of Hospital Accreditation on the Number of Occurrence Variance Report or Incident Reports. International Journal of Business, Management and Allied Sciences (IJBMAS), 5(1), 15-19.

[17] Shaikh, Z. M. (2018). A Comparative Study on Laboratory and Blood Bank Performance by Using the Quality Indicators. International Journal of Business, Management and Allied Sciences (IJBMAS), 5(1), $1-8$.

[18] Shaikh, Z. M., Al-Omari, A., \& Ahmed, A. (2018). The impact of CBAHI accreditation on critical care unit outcome quality measures: a case study. IJHS, 8(7), 394- 407.

[19] Shaikh, Z. M., Al-Omari, A., \& Ahmed, A. (2018). The Impact of Planetree Certification on a Nationally and Internationally Accredited Healthcare Facility and its Services. RESEARCH REVIEW International Journal of Multidisciplinary, 3(8), 318-332.

[20] Safeer Ahmad, Jitendra Singh, Mohammad Arif Kamal, and Zubair Mujeeb Shaikh, (2020). PersonCentered Care Design with Reference to Healthcare Outcomes in Saudi Arabia: An Overview. American Journal of Civil Engineering and Architecture, 8(3), 91-96.

[21] Gazala Khan (2020). The Impact of Healthcare Accreditation on Patients' Satisfaction: A Literature Review. International Journal of Health Sciences and Pharmacy (IJHSP), 4(1), 49-56.

[22] Shaikh, Zuber Mujeeb. (2020). Religious and Cultural Aspects of Hand Hygiene in Healthcare Accreditations: A Review of Literature. International Journal of Health Sciences and Pharmacy (IJHSP), 4(1), 40-48.

[23] Shaikh, Zuber Mujeeb. (2020). A Case Study on Recall of used Scopes in the Endoscopy Department by using a Failure Mode \& Effect Analysis (FMEA) Proactive Risk Management. International Journal of Health Sciences and Pharmacy (IJHSP), 4(1), 13-24. 
[24] Zuber Mujeeb Shaikh. (2019). The Impact of Hospital Accreditation on the Patients' Experience of Hemodialysis Department: A Case Study. International Journal of Health Sciences and Pharmacy (IJHSP), 3(1), 31-39.

[25] Zuber Mujeeb Shaikh. (2019). The Impact of Hospital Accreditation on the Patients' Experience of Emergency Department: A Case Study. RESEARCH REVIEW International Journal of Multidisciplinary, 4(2), 803-810.

[26] Shaikh, Z. M. (2016). The impact of hospital accreditation on the ambulance services satisfaction. International Journal of Emerging Research in Management \& Technology (IJERMT), 5(12), 76-84.

[27] Shaikh, Z. (2017). The Impact of Hospital Accreditation on the Patient's Satisfaction of Physiotherapy Department Services. International Journal of Business, Management and Allied Sciences (IJBMAS), 4(4), 143-154.

[28] Shaikh, Z. M. (2017). The Impact of Hospital Accreditation on the Patients Satisfaction of Dietary Services. International Journal of Business, Management and Allied Sciences (IJBMAS), 4(4), 1-12.

[29] Shaikh, Z. M. (2017). The Impact of Hospital Accreditation on the Patients Satisfaction of Laboratory Department Services. International Journal of Business, Management and Allied Sciences (IJBMAS), 4(2), 4277-4289.

[30] Shaikh, Z. M. (2017). The Impact of Hospital Accreditation on the Patients Satisfaction of Emergency Department Services. International Journal of Business, Management and Allied Sciences (IJBMAS), $4(3), 4330-4339$.

[31] Shaikh, Z. M. (2017). The Impact of Hospital Accreditation on the Patient Satisfaction of In-Patient Department Services. International Journal of Emerging Research in Management \& Technology (IJERMT), 6(8), 368-383.

[32] Shaikh, Z. M. (2017). The Impact of Hospital Accreditation on the Patients Satisfaction of Haemodialysis Department Services. International Journal of Emerging Research in Management \& Technology (IJERMT), 6(8), 384-392.

[33] Shaikh, Z. M. (2017). The Impact of Hospital Accreditation on the Patients Satisfaction of Radiology Department Services. International Journal of Business, Management and Allied Sciences (IJBMAS), 4(1), 4120-4130.

[34] Shaikh, Z. M. (2017). The Impact of Hospital Accreditation on the Patient's Satisfaction of Pharmacy Department Services. International Journal of Business, Management and Allied Sciences, 4(4), 189199.

[35] Shaikh, Z. M. (2017). The Impact of Hospital Accreditation on the Patients Satisfaction of Out-Patient Department Services. International Journal of Business, Management and Allied Sciences (IJBMAS), 4(3), 4384-4398.

[36] Zuber Mujeeb Shaikh (2018). Impact of National Accreditation on the Patients' Experience of Ambulance Services: A Case Study. RESEARCH REVIEW International Journal of Multidisciplinary, 3(08), 177-181.

[37] Zuber Mujeeb Shaikh. (2019). The Impact of Hospital Accreditation on the Patients' Experience of Hemodialysis Department: A Case Study. International Journal of Health Sciences and Pharmacy (IJHSP), 3(1), 31-39.

[38] Zuber Mujeeb Shaikh. (2019). The Impact of Hospital Accreditation on the Patients' Experience of Emergency Department: A Case Study. RESEARCH REVIEW International Journal of Multidisciplinary, 4(2), 803-810.

[39] Shaikh, Z. M. (2017). The Impact of Hospital Accreditation on the Completeness of Personnel Files in Human Resource Department. International Journal of Business, Management and Allied Sciences (IJBMAS), 4(4), 236-244. 
[40] Shaikh, Z. M. (2018). The Impact of Hospital Accreditation on the Number of Occurrence Variance Report or Incident Reports. International Journal of Business, Management and Allied Sciences (IJBMAS), 5(1), 15-19.

[41] Shaikh, Z. M. (2018). A Comparative Study on Laboratory and Blood Bank Performance by Using the Quality Indicators. International Journal of Business, Management and Allied Sciences (IJBMAS), 5(1), $1-8$.

[42] Shaikh, Z. M., Al-Omari, A., \& Ahmed, A. (2018). The impact of CBAHI accreditation on critical care unit outcome quality measures: a case study. IJHS, 8(7), 394- 407.

[43] Shaikh, Z. M., Al-Omari, A., \& Ahmed, A. (2018). The Impact of Planetree Certification on a Nationally and Internationally Accredited Healthcare Facility and its Services. RESEARCH REVIEW International Journal of Multidisciplinary, 3(8), 318-332.

[44] Shaikh, Z., Al-Towyan, S., \& Khan, G. (2016). Critical Analysis of Patient and Family Education in JCI Accreditation and CBAHI Standards for Hospitals. International Journal of Research in Business Management (IMPACT: IJRBM), 4(3), 29-38.

[45] Shaikh, Z., Al-Towyan, S., \& Khan, G. (2016). Critical Analysis of Staff Qualifications and Education Standards in JCI and Medical Staff \& Staffing Management Standards in DNV Accreditation for Hospitals. International Journal of Research in Business Management (IMPACT: IJRBM), 4(3), 61-70.

[46] Shaikh, Z., Al-Towyan, S., \& Khan, G. (2016). Critical Analysis of International Patient Safety Goals Standards in JCI Accreditation and CBAHI Standards for Hospitals. International Journal of Research in Business Management (IMPACT: IJRBM), 4(3), 71-78.

[47] Shaikh, Zuber. (2017). Critical Analysis of Patient and Family Rights in JCI Accreditation and CBAHI Standards for Hospitals. International Journal of Emerging Research in Management \& Technology (IJERMT), 6(7), 324-330.

[48] Shaikh, Zuber Mujeeb. (2020). A Case Study on Recall of used Scopes in the Endoscopy Department by using a Failure Mode \& Effect Analysis (FMEA) Proactive Risk Management. International Journal of Health Sciences and Pharmacy (IJHSP), 4(1), 13-24.

[49] Shaikh, Zuber Mujeeb. (2020). Religious and Cultural Aspects of Hand Hygiene in Healthcare Accreditations: A Review of Literature. International Journal of Health Sciences and Pharmacy (IJHSP), 4(1), 40-48.

$* * * * * * * * * * *$ 